\title{
The PCSS communication system based on the method of successive contrast
}

\author{
Bei-Ming Li, Shu-Heng Qi, Jun-Wei Qi and Cheng-Quan An \\ College of information and Communication Engineering, Harbin Engineering University \\ Harbin, China \\ E-mail: libeiming@hrbeu.edu.cn
}

\begin{abstract}
Parallel Combinatory Spread Spectrum (PC/SS) communication system has higher data transmission capability and bandwidth efficiency than conventional Spread Spectrum (SS) communication system. Meanwhile, PC/SS system must correctly select all maximal $r$ PN sequences from $M$ PN correlations, otherwise it will cause a lot of error data. The existing algorithm has no ability of detecting and correcting a random misjudging sequence. To solve this problem, PCSS communication system based on the method of successive contrast (SC/PC/SS) is proposed in this paper and performances are analyzed. The SC/PC/SS system can detect and correct a sequence error. Theoretical analysis and simulation results show that the BER performance of the SC/PC/SS system is lower than conventional PC/SS system in the same situation. Without additional hardware and slightly increase in complexity, it can improve the communication environment of $1 \mathrm{~dB}$.
\end{abstract}

Keywords: SS;PC/SS; Successive Contrast; Anti-Jamming.

\section{Introduction}

The parallel combination spread spectrum communication has the higher ability of information transmission and the band utilization rate[1]. According to the data-sequence mapping algorithm of PC/SS system, it will select $r$ sequences from alternative $\mathrm{PN}$ sequences to overlay and transmit in the way of parallel combination[2]. When receiving, PC/SS system must ensure all $r$ PN sequences selected decision correct, otherwise a number of error data will be received[3].

In the concerned of the problem above, the SC/PC/SS system is proposed in this paper, and the system has the ability to detect and correct one random misjudging sequence. In this paper, the fundamental principle of successive contrast is introduced firstly, then a comparison between the proposed method and conventional PC/SS is made in BER performance. In the end, the significant simulation results of successive contrast prove higher antijamming performance of PC/SS communication system. 


\section{Principle of the Method of Successive Contrast}

Transmitter and receiver configurations of the SC/PC/SS system are displayed in Fig.1 and Fig. 2 Compared with conventional PC/SS system[4], the additional section is as follows: In the receiver, after the decision of $r$ maximum sequences, the received information subtract the selected PN sequences successively and contrast with the remaining sequences. By detecting selected PN sequences, the more reliable sequences will be inverse mapping so that the data is correct.

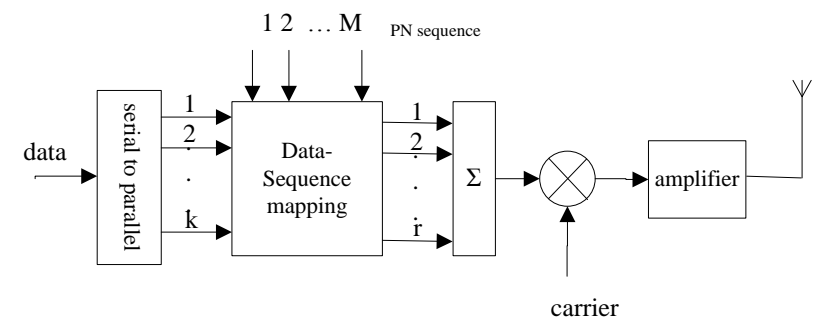

Fig. 1 Transmitter model of SC/PC/SS system

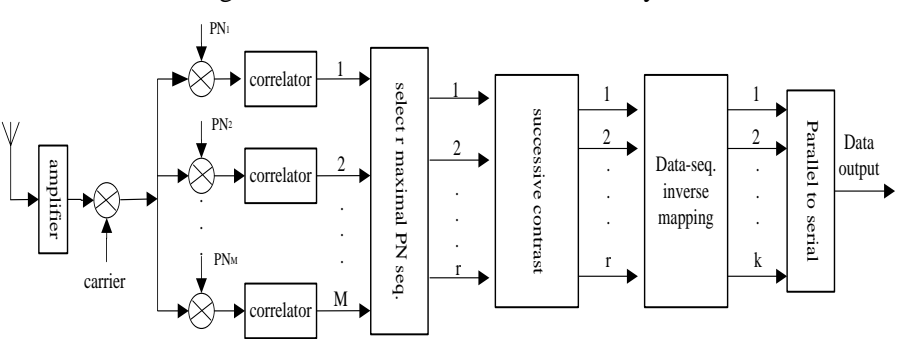

Fig. 2 Receiver model of SC/PC/SS system

Assume that the carrier that come from transmitter and reciever is synchronous, after demodulation, receive signals turns into composite signal which the range of the PC/SS signal and the noise normalized, $S(t)=\operatorname{Re} c(t)+N(t)$, $S(t)$ stands for composite signal. $\operatorname{Re} c(t)$ represents the received signal. $N(t)$ stands for noise and interference.

In the receiver, $r$ maximal sequences are selected from $M$ sequences (for instance, $M=16, r=3$ )and they are sorted from big to small such as $P N_{i}, P N_{j}, P N_{k}$. We set $P N_{i}, P N_{j}, P N_{k}$ as the initial sequence vector and define the detection vector $C_{0}$.The corresponding detection vector is 1 when a sequence appear. As A result, we can get the initial sequence vector $A=\left[P N_{i}, P N_{j}, P N_{k}\right]$ and the initial detection vector $C_{0}=[1,1,1]$, which represent the selected sequence appear once. If the received signal contains one of the sentenced sequence, this sequence is subtracted by $S(t)$, and then related to the local sequences. The related value of this sequence decreased significantly and the correlation value 
of the other two sequences is still large. On the contrary, the initial sentenced sequences may be misjudged if the correlation value of a new sequence is very large. Then, we detect that if the sentenced sequences are correct. Specific steps as follows:

(1) $S(t)-P N_{i}$ related to the local sequences and select 2 maximal sequences. We set detection vector $C_{1}=[0,0,1]$ and sequence vector $A 1=A$ if the selected sequences are $P N_{j}$ and $P N_{k}$, otherwise we extend sequence vector $A_{1}=\left[A, P N_{x}\right]$ and calculate detection vector $C_{1}$;

(2) $S(t)-P N_{j}$ related to the local sequences and select 2 maximal sequences. We set sequence vector $A_{2}=A_{1}$ and calculate detection vector $C_{2}$ if the selected sequences are $P N_{i}$ and $P N_{k}$, otherwise we extend sequence vector $A_{2}=\left[A_{1}, P N_{y}\right]$ and calculate detection vector $C_{2}$;

(3) $S(t)-P N_{k}$ related to the local sequences and select 2 maximal sequences. We set sequence vector $A_{3}=A_{2}$ and calculate detection vector $C_{3}$ if the selected sequences are $P N_{i}$ and $P N_{j}$, otherwise we extend sequence vector $A_{3}=\left[A_{2}, P N_{z}\right]$ and calculate detection vector $C_{3}$;

(4)Calculate detection vector $C=C_{0}+C_{1}+C_{2}+C_{3}$.if $C=[3,3,3]$, correct selected sequences and data-sequence inverse mapping, otherwise put the forth sequence instead of the third one and data-sequence inverse mapping.

\section{Error Rate Analysis}

The receiver of PC/SS system selects the maximal $r$ sequences from $M$ correlation output ${ }^{[5]}$. When $m 1 \in\left\{i_{1}, i_{2}, \ldots i_{r}\right\}, m 2 \notin\left\{i_{1}, i_{2}, \ldots i_{r}\right\},\left\{i_{1}, i_{2}, \ldots i_{r}\right\} \in\{1,2 \ldots M\}$, the correct decision probability for identifying an active PN sequence is expressed by $^{[6]}$

$$
\begin{aligned}
P_{r_{m}} & =\left\{\left|V_{m_{1}}\right|>0,\left|V_{m_{1}}\right|>\left|V_{m_{2}}\right|\right\} \\
& =\frac{1}{\sqrt{2 \pi}} \int_{-\sqrt{h}}^{\infty} e^{-x^{2} / 2}\left[1-\operatorname{erfc}\left(\frac{x+\sqrt{h}}{\sqrt{2}}\right)\right]^{(M-r)} d x
\end{aligned}
$$

Where $h=\sqrt{2 P T / N_{0}}, \operatorname{erfc}($.$) is complementary error function.$

The decision of each transmission sequence is independent and equal probability. Conventional PC/SS system must estimate correctly maximal $r$ sequences from $M$ correlation outputs, otherwise most information is error. The correct decision probability of symbol is obtained by ${ }^{[7]}: P_{r}=\left(P_{r_{m}}\right)^{r}$ Thus, the BER of conventional PC/SS system is computed by

$$
P_{e}=1-P_{r_{m}}^{r}
$$


The following events are defined: $A$ :there is a sequence misjudged from maximal $\mathrm{r}$ sequences when receiving; $B$ : the $r^{\text {th }}$ sequence misjudged and the $(r+1)^{t h}$ sequence is correct. Thus the probability of $A$ and $B$ can be expressed by

$$
\begin{aligned}
& P(A)=P_{r_{m}}^{r-1} \times\left(1-P_{r_{m}}\right) \times \frac{1}{M-r} \times\left(1-\frac{1}{M-r}\right)^{M-r-1} \\
& P(B \mid A)=P_{r_{m}}^{r-1} \times \frac{1}{M-r} \times\left(1-P_{r_{m}}\right) \times \frac{1}{r} \times\left(1-P_{r_{m}}\right)
\end{aligned}
$$

The event is defined: $C$ : there is a sequence misjudged from maximal $r$ sequences, meanwhile, the $r^{\text {th }}$ sequence misjudged and the $(r+1)^{\text {th }}$ sequence is correct. It is obvious seen that $C$ is the event that $A$ and $B$ occur at the same time, its probability can be expressed by

$$
P(C)=P(A B)
$$

According to the conditional probability formula, it can be transformed into

$$
P(C)=P(A) P(B \mid A)
$$

According to the definition, $P(C)$ is the improving probability of conventional system. Therefore, the BER of improved PC/SS system can be computed by

$$
P_{e}^{\prime}=P_{e}-P(C)=P_{e}-P(A) P(B \mid A)
$$

\section{Numerical Results}

We consider a particular situation in which the number of assigned PN sequences $M$, and the number of the sequences $r$ are given to be constant. But the number of the sequences length are different ( $L=127, L=255, L=511)$. The BER against the SNR per information bit is shown in Fig. 3 and BER comparison between different length sequences of SC/PC/SS and PC/SS in Fig.4.

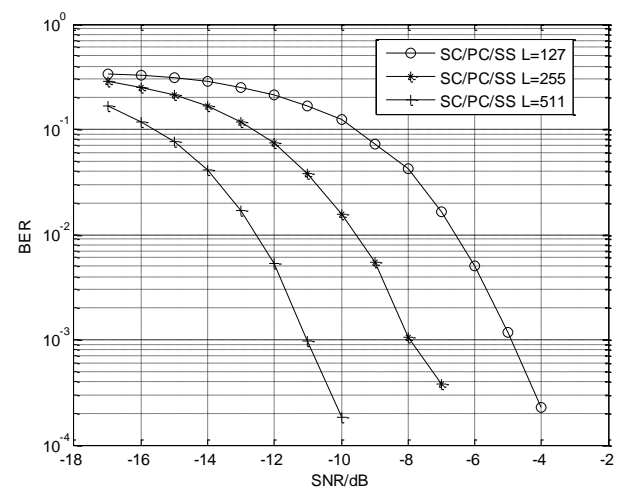

Fig. 3 BER comparison between different length sequences of SC/PC/SS ( $M=16, r=3$ ) 


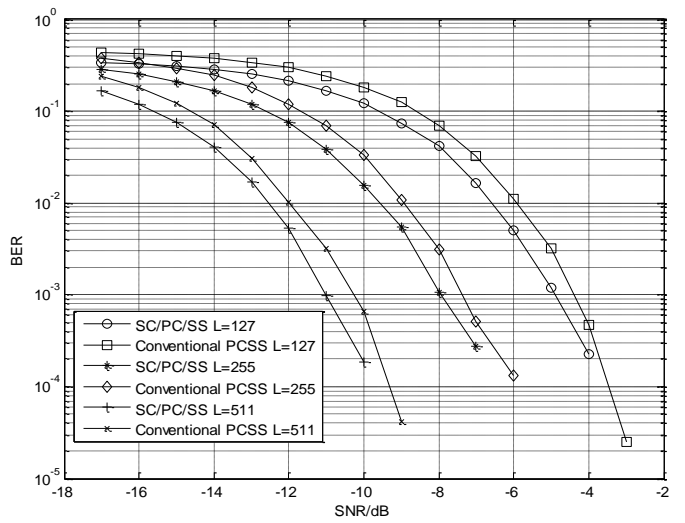

Fig. 4 BER comparison between different length sequences of SC/PC/SS and PC/SS( $M=16, r=3$ )

Fig. 3 and Fig. 4 shows that under the same SNR conditions, the longer of the sequence, the lower BER of the system and the higher quality of the communication. As we all know, in case of guaranteeing the reliability of the communication, the longer the sequence, the lower information transmission rate. In this way, we can improve the information transmission rate. It is obvious seen that performance of SC/PC/SS system is improved. This method is suitable for long sequence communication environment.

\section{Conclusion}

The symbol and average bit error rate performance of SC/PC/SS system are investigated. By using SC/PC/SS system, the decision error probability can be decreased during one PN period. The BER can be improved by applying the method of successive contrast comparing with the conventional PC/SS systems in the case that the number of assigned $\mathrm{PN}$ sequence and information bit rate are constant. SC/PC/SS system has been proposed to accomplish high-data transmission and low error rate performance. Furthermore, SC/PC/SS system has excellent secret property that would have wildly application in civil and military communication.

\section{Acknowledgments}

This research has been supported by International Science and Technology Cooperation Program of China (2014 DFR10240), Hei Long Jiang Postdoctoral Foundation (LBH-Z14066), Science Foundation of Heilongjiang Province 
QC2015075, and Fundamental Research Funds for the Central Universities GK2080260146.

\section{References}

1. J.K. Zhu, S. Shigenobu, and M. Gen, "Proposal of parallel combinatory spread spectrum communication system," Trans. on IEICE, J74-B II (5), pp.207-214, 1991.

2. J.K. Zhu, "Properties and application of parallel combinatory SS communication system, " IEEE Second Intemational Symposium on Spread Spectrum Techniques and Applications, vol.12, no.1, pp.227-230, 1992.

3. M. Wang, L.L. Guo, and Q. Yu, “Analysis of Coded Parallel Combinatory Spread Spectrum Comunication System Based on Improved r-combinatory Mapping," Ship Electronic Engineering,vol.10, no.28, pp.78-81, 2008.

4. X.D. Qi, Z.G. Sun, and Q. Luo, "Improved detection algorithm for parallel combinatory spread spectrum signals," Journal of Harbin Engineering University, vol. 30, no.2, pp.215-218, 2009.

5. K. Zhang, X.Q. Zhao, and Y. Zhang, "Optimized Selection of Spread Spectrum Sequence for Parallel Combinatory Spread Spectrum Communication," Communications Technology, vol.1, no.45,pp.115-118, 2012.

6. L.L. Guo, Q. Yi, and B.M. Li,"Parallel Combinatory Spectrum Communication System Based on r-Combinatory and the Study of PN Sequences," Radio Communications Technology, vol.33, no.4,pp.25-27, 2007.

7. GUO Li-li, LI Bei-ming. BER performance of complex parallel combinatory spread spectrum communication system [C]// Wireless Communications, Networking and Mobile Computing, 2007: 1365-1368. 